\title{
RELACION ENTRE DISPLASIA SEVERA Y CARCINOMA IN SITU DEL CUELLO UTERINO
}

\author{
Dr. Alvaro Fonnegra M.* \\ Dr. Rubén Darío Guzmán A.** \\ Dr. Jaime Cantillo G.***
}

Universidad Nacional de Colombia - Facultad de Medicina - Bogotá.

El presente trabajo tiene por objeto destacar la importancia que tiene la relación carcinoma in situ y la displasia severa.

Con el avance de los estudios citohistológicos, se le ha dado últimamente mayor precisión al concepto de displasia, englobando en este término una serie de transformaciones y modificaciones patológicas cervicales, que antes eran vagas, dispersas y se prestaban a controversias. Hoy en día se acepta como Displasia, la presencia de células epiteliales anormales consideradas aisladamente; no se trata de una sencilla hiperplasia. También es conocida con los nombres de anaplasia, lesión límite o discutible, metaplasia pre-cancerosa, lesión equívoca, hiperplasia epitelial de la capa de células basales, hiperplasia epitelial atípica. Sea cual fuere el nombre dado a la displasia, la importancia de descubrirla estriba en el supuesto papel que tiene en la génesis del carcinoma in situ del cérvix; así Koss y cols. (1) observaron que más del $40 \%$ de estas lesiones presentaban progreso a carcinoma in situ y que el $38 \%$, desaparecían. Stern y Neely (2), informan en un estudio realiza- do por ellos que el $12 \%$ de los casos de displasia progresaron a carcinoma in situ; un $40 \%$ experimentaron regresión al estado normal y un $40 \%$ continuaron siendo displasias.

Scott y Ballard (3) entre 221 mujeres que presentaban displasia comprobada histológicamente, en 48,9\% ulteriormente se comprobó que no había secuelas; en $43.9 \%$ había displasia persistente cuando se vigilaron por última vez; en $6.3 \%$ se demostró posteriormente $\mathrm{Ca}$. in situ y el $0.9 \%$ resultó por último $\mathrm{Ca}$. invasivo. Según Fluhmann (4), cuando se sigue mediante biopsias repetidas durante períodos de uno a cuatro años, se observa que la lesión desaparece aparentemente por regresión o por extirpación mediante biopsias, en un 20 a $40 \%$; persiste invariable en un 20 a $48 \%$ y por último, en un 3.8 a $17 \%$ ya seguido de Ca. in situ. Se-

* Profesor Asociado. Departamento de Obstetricia y Ginecología, Facultad de Medicina. Universidad Nacional de Colombia.

** Profesor Asociado. Departamento de Obstetricia y Ginecología, Facultad de Medicina. Universidad Nacional de Colombia.

*** Profesor Asistente. Departamento de Patología, Facultad de Medicina. Universidad Nacional de Colombia. 
gún McKay (5) y cols., se encuentra asociada al $\mathrm{Ca}$. in situ en el $32.5 \%$. Reagan (6) afirma que en $82 \%$ de los casos de carcinoma que estudió había displasia concomitante. Baker y Haws (7) y May (8), han encontrado hasta 82 casos de displasias entre un centenar de casos de carcinoma in situ. Fundadas en estas geenralizaciones, parece justificado aceptar la displasia como una lesión que guarda una relación con el $\mathrm{Ca}$. in situ, muy similar al del $\mathrm{Ca}$. in situ, respecto al Ca. invasor.

El diagnóstico no siempre es claro y un anatomopatólogo puede considerar una determinada lesión como displasia y otro como $\mathrm{Ca}$. in situ.

Según Reagen y Wentz (9), existen pruebas de que esta entidad es menos frecuente en la no embarazada y de que aumenta la frecuencia según la paridad. Los datos epidemiológicos son semejantes a los observados para el $\mathrm{Ca}$. de cérvix. Según ellos mismos conceptúan que cuando se observa aisladamente la reacción displásica puede ocurrir durante el período de actividad sexual; se ha observado después de la menopausia sólo cuando hay estrógenos; ello sugiere que los estrógenos pueden ser factor que participa en la aparición de estos cambios. En nuestro medio presentamos 31 casos de $\mathrm{Ca}$. in situ concomitantes con displasias severas, extractados de 105 historias clínicas de Ca. in situ, diagnosticados y tratados en el servicio de Ginecología y Obstetricia del Hospital San Juan de Dios y del Instituto Materno Infantil "Concepción Villaveces de Acosta" de Bogotá, en un lapso de 11 años, lo que dá un porcentaje de concomitancia, Ca. In-situ-displasia de 32,55\%, demostrado, como lo veremos a lo largo del estudio de dichos casos.
Por otra parte fueron excluídos de este estudio 14 casos, 12 de Ca. infiltrante y 2 de $\mathrm{Ca}$. microinvasivo, concomitantes con $\mathrm{Ca}$. in situ, que no son motivo de este trabajo, pero que pone de manifiesto la importancia de la multifocalidad de estas lesiones encontradas en el cono.

De acuerdo con lo anterior, presentamos los Cuadros con los casos clínicos y los datos más importantes así :

1. Edad, motivo de consulta, estado del cuello, gravidez, citología, biopsia, conización, tumor residual post-cono, histerectomía abdominal ampliada, tumor residual post-histerectomía, controles.

1 ㅇ.

No DE ORDEN

\begin{tabular}{lcc}
\hline & Casos & $\%$ \\
\hline (Enviados de Profamilia) & 16 & 51,60 \\
Consulta Externa general & 15 & 48,40 \\
\hline
\end{tabular}

\section{2 .}

EDAD

\begin{tabular}{lrlr}
\hline & & Casos & $\%$ \\
\hline De 20 a 30 años & 6 & 19,36 \\
Más de 30 a 40 años & 17 & 54,84 \\
Más de 40 a 50 años & 6 & 19,36 \\
Más de 50 años & 2 & 6,44 \\
\hline
\end{tabular}

3 ․

PARIDAD

\begin{tabular}{lcr}
\hline & Casos & $\%$ \\
\hline Nuligestante & 1 & 3,23 \\
1 a 2 partos & 4 & 12,90 \\
Más de 2 a 5 partos & 9 & 29,03 \\
Más de 5 partos & 17 & 54,84 \\
\hline
\end{tabular}


$4:$

MOTIVO DE CONSULTA

\begin{tabular}{|c|c|c|c|}
\hline & & Casos & $\%$ \\
\hline Asintomática & $z$ & 14 & 45,16 \\
\hline Hemorragia genital & & 5 & 16,13 \\
\hline Flujo genital & & 9 & 29,03 \\
\hline Otros & & 3 & 9,68 \\
\hline
\end{tabular}

\section{5 웅}

ESTADO DEL CUELLO

\begin{tabular}{lcc}
\hline & Casos & $\%$ \\
\hline Erosión & 24 & 77,42 \\
Aparentemente sano & 7 & 22,58 \\
\hline
\end{tabular}

6:

CITOLOGIA

\begin{tabular}{lrrr}
\hline & & Casos & \multicolumn{1}{c}{$\%$} \\
\hline A & 5 & 4 & 12,90 \\
$\mathrm{~A}$ & 4 & 19 & 61,29 \\
$\mathrm{~A}$ & 3 & 7 & 22,58 \\
$\mathrm{~A}$ & 2 & 1 & 3,23 \\
\hline
\end{tabular}

7ㅇ.

BIOPSIA

\begin{tabular}{lcc}
\hline & Casos & $\%$ \\
\hline Ca in situ + Displasia severa & 21 & 67,74 \\
Displasia severa & 10 & 32,26 \\
\hline
\end{tabular}

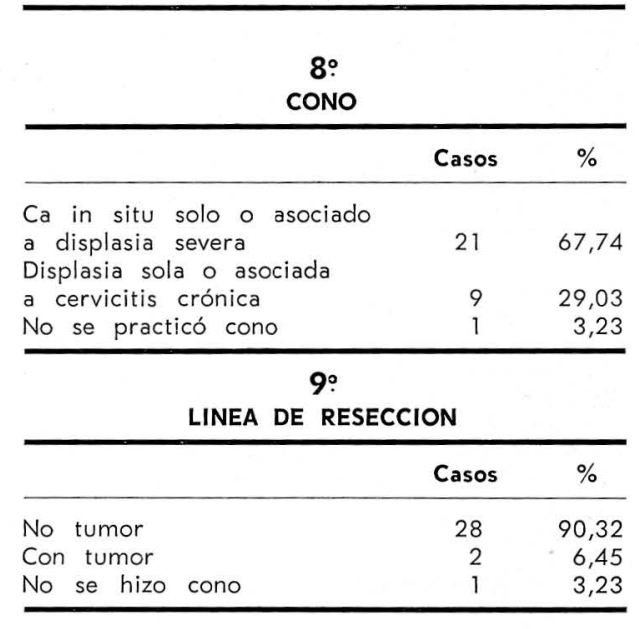

10 :

HISTERECTOMIA AMPLIADA

\begin{tabular}{lrr}
\hline & Casos & \multicolumn{1}{c}{$\%$} \\
\hline Se practicó & 19 & 61,29 \\
Con S. O. B. & 1 & 3,23 \\
No se practicó & 11 & 35,48 \\
\hline
\end{tabular}

11 :

COMPLICACIONES URINARIAS DE LA HISTERECTOMIA AMPLIADA

\begin{tabular}{lrr}
\hline & Casos & \multicolumn{1}{c}{$\%$} \\
\hline Fístulas uretero-vaginal & 2 & 6,45 \\
Sin complicaciones & 18 & 58,07 \\
No se practicó & 11 & 35,48 \\
\hline
\end{tabular}

$12^{\circ}$

LESION RESIDUAL EN EL UTERO

\begin{tabular}{lrr}
\hline & Casos & $\%$ \\
\hline Sin lesión & 14 & 45,17 \\
Displasia severa & 5 & 16,12 \\
Adenomiosis & 1 & 3,23 \\
\hline
\end{tabular}

13 ?

CONTROLES

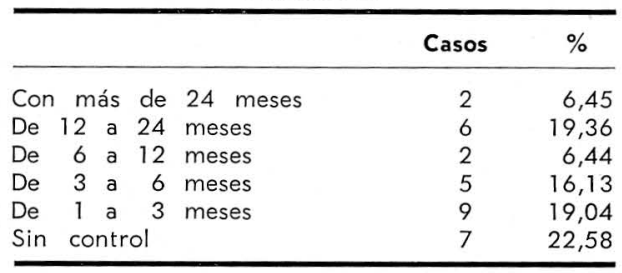

Analizándolos, podemos hacer las siguientes consideraciones:

1. La edad promedio fue de 30 a 40 años; tenemos que anotar que figuran tres pacientes de 22, 25 y 28 años respectivamente, lo que demuestra que estas lesiones aunque raras en estas edades, no son excepcionales.

2. En la mayor parte de las pacientes, $45,28 \%$, las lesiones fueron en- 
contradas por citología vaginal de rutina o remitidas de pro-familia, (campaña de planificación familiar), es decir, asintomáticas en cuanto a patología de cuello se trataba; en un $33,33 \%$ las enfermas consultaron por flujo, consulta muy común en nuestras pacientes hospitalarias y síntoma que puede corresponder a un buen número de entidades. 9,4\% consultaron por problemas extragenitales y sólamente un $12,6 \%$ se quejaron de hemorragia genital, síntoma típicamente relacionado con $\mathrm{Ca}$. de cuello uterino. Este último análisis demuestra que tanto la displasia con el Ca. in situ son en la mayor parte de los casos asintomáticos.

3a En 24 pacientes (73\%), existía erosión de cuello y en el resto, o sean 7 pacientes ( $27 \%$ ), éste se encontró aparentemente sano. Esto indica la importancia de la inspección directa del cuello uterino en todo examen ginecológico y además la toma rutinaria de la citología.

Las biopsias y conizaciones confirmaron el diagnóstico de $\mathrm{Ca}$. in situ y de displasia severa y en no pocos casos, multifocalidad de ambas lesiones. La parte más importante del trabajo y lo que aquí se prueba, es la relación íntima de las dos entidades, demostrada a lo largo del estudio en cada paso de la biopsia, como en histerectomía abdominal ampliada. Así tenemos que en 16 casos $(51,6 \%)$, hubo $\mathrm{Ca}$. in situ en la biopsia y en el cono apareció displasia severa y al contrario, en 9 casos, se evidenció displasia en la biopsia y $\mathrm{Ca}$. in Situ en el cono. Por otra parte, en 5 casos $(16,1 \%)$, no se evidenció displasia ni en la biopsia ni en el cono, en cambio apareció en la pieza quirúrgica de la histerectomía abdominal ampliada, último paso en el tratamiento del Ca. in situ. Esto demues- tra una vez más, la multifocalidad de estas lesiones.

Hay varios casos que requieren comentarios especiales, a saber:

CASO No 1. En el cual la biopsia y el cono demostraron $\mathrm{Ca}$. in situ, pero hubo tumor residual en este último y al practicar la histerectomía ampliada apareció la displasia. Este caso ha debido ser reconizado antes de la histerectomía ampliada.

. CASO No 4. Enferma con el diagnóstico de Ca. in situ y displasia en la conización, se le hizo histerectomía ampliada con SOB, con ovarios normales. Tenemos que criticar esta conducta de extirpar gónadas sanas en este tipo de cirugía. Sólamente se justifica extraer ovarios cuando hay patología macroscópica en dichos órganos, concomitante con el $\mathrm{Ca}$. in situ.

CASO No 29. Paciente de 35 años de edad G8. P7. A1., citología A4. con prolapso genital de tercer grado y displasia severa en la biopsia, a quien no se le practicó sino directamente operación de Manchester con amputación de cuello y corrección de celes, evidenciándose en el cuello amputado un $\mathrm{Ca}$. in situ con invasión glandular. La conización no ha debido ser omitida, como paso previo a la corrección de los celes.

CASOS 1 y 13. Pacientes con carcinoma in situ y displasia severa que se trataron con histerectomía ampliada, presentando como complicación, sendas fístulas uterevovaginales derechas.

CASOS Nos. 12, 16 y 23. Pacientes de 25, 22 y 28 años de edad, respectivamente, muy jóvenes las dos primeras y primigestante la tercera, con Ca. in situ en la biopsia y displasia moderada y severa en el cono, a quienes se dejó en fase de conización como tratamiento, debido a la edad. Hay que recalcar que esta conducta es excepcional, tomada en mujeres jóvenes, que sean de fácil control, nulíparas o que deseen una nueva maternidad. El tratamiento del Ca. in situ concomitante o no con displasia severa, debe ser en la casi totalidad de los casos, la histerectomía ampliada (histerectomía total con tercio superior de vagina).,

CASOS Nos. 27, 28 y 31. Enfermas jóvenes (29, 27 y 29 años respectivamente), multigestantes, que pidieron fueran intervenidas por histerectomías ampliadas.

CASO No 11. Paciente de 35 años de edad GO. PO. con Ca. in situ en la biopsia y dis- 
plasia severa en la conización, se considera un caso muy raro desde el punto de vista epidemiológico, nuligestante y con $\mathrm{Ca}$.

CASOS Nos. 8, 9, 14, 19 y 25. Pacientes de $34,35,33,38$ y 40 años de edad respectivamente, con diagnóstico de $\mathrm{Ca}$. in situ en la biopsia y displasia en el cono, las cuatro primeras multigestantes y la última G1. P1., quienes rehusaron la histerectomía ampliada como tratamiento final.

Como se ve, esta alta cifra de concomitancia de las dos entidades, pone de manifiesto la importancia de estudiar exhaustivamente la paciente que presente displasia en algunos de los pasos obligados en la metodología diagnóstica llevada a cabo en patología de cuello uterino, (citología vaginal seriada, biopsia, cono).

\section{Controles}

Una de las fallas de la asistencia social de nuestros hospitales, es la búsqueda de las pacientes operadas de enfermedades que necesiten controles rigurosos y una de éstas, es el cáncer, motivo por el cual no se ha podido organizar la asistencia regular y numerosa de toda esa clientela hospitalaria. En consecuencia, las cifras de controles, aunque han mejorado, todavía continúan siendo muy pobres.

De acuerdo con lo anterior tenemos los siguientes datos: En un lapso de 4 años, tiempo en que se encontraron los casos de displasia y $\mathrm{Ca}$. in situ concomitante, se practicaron controles, así:

Dos (2) casos con más de 24 meses.

Seis (6) casos de 12 a 24 meses.

Dos (2) casos de 6 a 12 meses.

Cinco (5) casos de 3 a 6 meses.

Nueve (9) casos de 1 a 3 meses.

Siete (7) casos sin control.
Como se observa, todavía no ha habido el suficiente tiempo para apreciar resultados de supervivencia; sin embargo, en un trabajo futuro, podremos presentar casos con cinco o más años de controles. De todas formas, ninguno de los casos controla dos hasta el presente, ha presentado recurrencia, ni ha habido mortalidad.

\section{Resumen y conclusiones}

Se presenta un estudio de 31 casos de $\mathrm{Ca}$. in situ concomitantes con displasias severas, sobre un total de 105 casos de $\mathrm{Ca}$. in situ diagnosicados y tratados en el servicio de Ginecología y Obstetricia del Hospital San Juan de Dios y del Instituto Materno Infantil "Concepción Villaveces de Acosta", de Bogotá, en un lapso de 11 años; el porcentaje de concomitancia de las dos entidades es de $32.55 \%$.

Se hace la consideración de lo que es una displasia y su importancia histopatológica en la génesis del Ca. in situ de cuello uterino.

Además se analiza la metodología seguida, sobre edad, motivo de consulta, estado del cuello, gravidez, citología, biopsia, conización, tumor residual post-cono, histerectomía total ampliada, tumor residual post-histerectomía y controles.

\section{Summary and Conclusions}

Presentation of the study of $31 \mathrm{ca}$ ses of carcinoma in situ, concomitant with severe dysplasia, of a total of 105 cases of carcinoma in situ diagnosed and treated at the Gynecological and Obstetrics Ward of Hospital San Juan de Dios and of Instituto Materno Infantil "Concepción Villaveces de Acosta" in Bogotá, within a period of 11 years. The percentage of concomitance of the two events is 32.55 . 
Consideration is given to the definition of a dysplasia and its hystopathological importance $i$ nthe origin of carcinoma in situ at the cervix uteri.

The methodology followed is also analyzed with regards to age, reason for consultation, status of the cervix, pregnancy, cytology, biopsy, conization, residual post-cone tumour, enlarged total hysterectomy, residual post-hysterectomy tumour and control.

\section{BIBLIOGRAFIA}

1 KOSS L. G., STEWART, F. W., FOOTE, F. W., JORDAN, M. J., BEDER G. M. Y DAY, E.: Some Histological aspects of behavior of epidermoid carcinoma in situ and related lesions of the uterine cervix. Cáncer. 10: $1160,1963$.
2 STERN, E. y NEELY, P. M.: Carcinoma and dysplasia of the cervix. Acta Cytol. 7: 357, 1963.

3 SCOTT, R. B. y BALLARD, L. A.: Problems of cervical biopsy. Ann. N. Y. Acad. Sci., $97:$ 707, 1962.

4 FLUHMANN C. DRODERIC. EI cuelo uterino y sus enfermedades. Salvat Editores. Primera edición. 1963.

5 MaCKAY, D. G., TERJANIAN, B., POSCYACHINDA, D., YOUNGE, P. A., y HERTIG A. T.: Obst. \& Gynec. 13, 2, 1959.

6 ECAGAN, J. W. ,DICK, D. J. y SCOTT. R. E.: Atypical hyperplasia of uterine cervix. Cancer, 8: 42, 1956.

7 BAKER, W. S. y HAWKS, B. L.: Am. J. Obst. \& Gynec. 73, 1266, 1957.

8 MAY, H. C.: Am. J. Obst. y Gynec. 76, $807,1958$.

9 REAGAN, JAMES W., WENTZ W. DUDD: Génesis del Carcinoma del Cuello Uterino. Clínicas Obstétricas y Ginecológicas. Dic. 1967. 